\title{
Gesucht - Gefunden: Vergemeinschaftungs- und Reproduktionsleistungen jüdischer Heiratsvermittler*innen
}

\author{
Sarah AFFENTRANGER ${ }^{1}$ \\ Universität Freiburg i. Ü.
}

\begin{abstract}
Jüdische Heiratsvermittler*innen übernehmen eine wichtige Vermittlerrolle in der traditionellen Partner*innensuche. Sie lassen sich analytisch zwischen dem Individuum, der Religion und dem Staat positionieren und ihre Arbeit hat sowohl auf der Mikro- als auch auf der Makroebene Auswirkungen. Der vorliegende Artikel geht der Frage nach, wie jüdische Heiratsvermittler*innen dazu beitragen, die transnationale Vergemeinschaftung des jüdischen Diaspora-Netzwerks aufrechtzuerhalten. Es wird aufgezeigt, dass jüdische Heiratsvermittler*innen einen wichtigen Beitrag für die Kontinuität des Judentums leisten, indem sie sowohl die Reproduktion jüdischer Personen wie auch die der jüdischen Diaspora und des Staates Israel sicherstellen. Das Beispiel jüdischer Heiratsvermittler*innen macht deutlich, wie gesellschaftliche Strukturen und Normen individuelles Verhalten beeinflussen und schliesslich einen für die Kontinuität der jüdischen Gemeinschaft existenziellen Prozess der sozialen Schliessung unterstützen. Es steht beispielhaft für Triebkräfte von Vergemeinschaftungsprozessen, welche auch in anderen religiösen Gruppierungen beobachtbar sind.

Schlüsselwörter: Traditionelles Judentum, Heirat, Reproduktion und soziale Konstruktion der Nation, Diaspora, soziale Schliessung
\end{abstract}

\section{Einleitung und Fragestellung}

Die Tatsache, dass es heute noch Juden gibt und es immer Juden geben wird, ist, dass wir das einzige Volk auf Erden sind, das es tatsächlich immer wieder gewusst hat, trotz aller Unwägbarkeiten, die wir hatten, [...] immer wieder innerhalb des eigenen Volkes, dieses Volk zu vermehren. Das ist zum grossen Teil durch die Shadchanim,

\footnotetext{
${ }^{1}$ Sarah Affentranger studiert Soziologie, Sozialarbeit und Sozialpolitik (MA) an der Universität Freiburg i.Ü. und ist Stv. Leiterin von DAS HAUS - ein sozialpädagogisch betreutes Wohnprogramm der Stiftung Equipe Volo (sarah.affentranger@unifr.ch).
} 
also durch die Heiratsvermittler [geschehen]. (José Weber; zit. nach Knüppel 2013: o.S.)

Ehevermittlung geniesst eine langjährige Tradition im Judentum. Mit Hilfe sogenannter Schadchen (Heiratsvermittler*innen) wird sichergestellt, dass Juden ${ }^{*},{ }^{2}$ die überall verstreut auf der Welt in Diaspora-Gemeinschaften leben, denen ihre Identität und Nationalität wichtig sind, eine $^{\star} n$ geeignete $n$ ebenfalls jüdische ${ }^{\star} n$ Partner $^{\star}$ in finden. Laut der Online-Partnervermittlungsagentur Simantov International steigt die Nachfrage nach professionellen Heiratsvermittler*innen im 21. Jahrhundert und geniesst dabei zunehmende Beliebtheit bei jüdischen Singles auf der ganzen Welt (vgl. Simantov International 2018). Da Heirat und Familiengründung im traditionellen orthodoxen Judentum als religiöse Verpflichtung angesehen werden, die Einhaltung des religiös endogamen Heiratsregimes oder die weiten Distanzen zwischen einzelnen Diaspora-Netzwerken die Partner*innensuche aber erschweren, geniesst die Assistenz bei ebendieser hohes Ansehen.

Heiratsvermittler*innen sind besonders interessant, da sie sich zwischen Religion (Judentum), Reproduktion (als gesellschaftlicher, kultureller Prozess) und dem Staat (matrilineare Vererbung der Religion und der israelischen Staatsbürgerschaft) positionieren. Auf der Mikroebene, wo Heiratsvermittler*innen im Normalfall für das persönliche Glück von Liebessuchenden verantwortlich sind, zielt im Fall der Schadchen, die Partner*innensuche auch auf die Stärkung der Gemeinschaft ab. Sie übernehmen die Aufgabe und die Verantwortung des Individuums, eine ${ }^{\star} n$ geeignete ${ }^{\star} n$ Partner ${ }^{\star}$ in $z u$ finden, um eine Familie zu gründen und auf einer damit verbundenen Makroebene den Fortbestand über weitere Generationen sicherzustellen. Schadchen verstehen ihre Arbeit als Reproduktion der jüdischen Gemeinschaft, indem sie die Kontinuität und die soziale Konstruktion der Nation sicherstellen. Es geht also um die Nationenbildung via Internet, wobei sich diese translokale ethno-religiöse Community (Diaspora) durch eine Verstärkung der In-Group-Solidarität und durch die praktizierte Endogamie auszeichnet.

Das oben aufgeführte Zitat des jüdischen Heiratsvermittlers José Weber greift das Forschungsinteresse des vorliegenden Artikels auf prägnante Weise auf: Um die Kontinuität einer Gesellschaft sicherzustellen, ist Reproduktion erforderlich. Die jüdische Gemeinschaft ist ein Beispiel einer translokalen Vergemeinschaftung, die nicht nur an einen territorial festgesetzten Staat gebunden ist, sondern sich als Nation in vielen Diaspora-Gemeinschaften organisiert. Jüdische Heiratsvermittler*innen nehmen in der Sicherung des Fortbestands der Gemeinschaft eine wichtige Funktion ein. Was genau als guter Match gilt, welche Rolle dabei die Heiratsvermittler*innen einnehmen und welche religiösen und staatlichen Normen die Partnerinnenfindung beeinflussen, sind Aspekte folgender Forschungsfrage: Wie und weshalb tragen jüdische Heiratsvermittler*innen zur Reproduktion der transnationalen Vergemeinschaftung des jüdischen Diaspora-Netzwerks bei?

Im ersten Teil des Beitrags wird der thematische Kontext der Forschungsfrage anhand der Leistungen der jüdischen Heiratsvermittler*innen hinsichtlich der Religion, der Reproduktion, sowie der Nation dokumentiert. Im nächsten Schritt werden relevante Netzwerktheorien thematisiert. Dabei werden Theorien zur Kreuzung sozialer Kreise sowie der relationalen

\footnotetext{
${ }^{2}$ Im vorliegenden Beitrag wird mittels Gender-Sternchen der genderneutralen Schreibweise auf jüdische Personen verwie-
} sen. 
Soziologie vorgestellt. Theorien zu sozialen Netzwerken unterstützen dabei die Einbindung in grössere gesellschaftliche Zusammenhänge. Dazu gehören Überlegungen zu Diasporas sowie zu Möglichkeiten und Auswirkungen digitaler Medien. Da die Fragestellung sowohl auf einer Mikro- wie auch auf einer Makroebene interessante Forschungsaspekte aufweist, wird stets Bezug auf beide Ebenen genommen und deren Wechselwirkungen thematisiert. Im Anschluss wird am Fallbeispiel der jüdischen Partnervermittlungsplattform Simantov International eine Synthese präsentiert. Im abschliessenden Fazit werden die wichtigsten Zusammenhänge und Wirkungen zusammengefasst.

\section{Endogame Heiratsregime zur Reproduktion der Nationalität}

Religiöse Endogamie ist im Fall der jüdischen Gemeinschaft analog zu anderen religiösen Gemeinschaften ein zentrales Postulat, da damit die Kontinuität der Gemeinschaft gesichert wird (vgl. Goldlust 2001). Ein jüdisch-orthodoxes Dekret besagt, dass ein Kind nur als jüdisch anerkannt wird, wenn seine Mutter Jüdin ist. Dementsprechend wird im traditionellen Judentum Heirat als religiöse Verpflichtung gesehen. Die religiöse Endogamie ist, trotz der möglichen grossen Distanzen zwischen den Diaspora-Gesellschaften, strikt einzuhalten (vgl. Peres, Meisels und Frank 1980). So sichert das religiös-endogame Heiratsregime des traditionellen Judentums die Reproduktion des Judentums und somit auch jene der Nation (vgl. Goldlust 2001).

Jüdisch-orthodoxe Alleinstehende sind gemäss Levitz einem besonderen Druck ausgeliefert. „Mating anxiety“ (Levitz; zit. nach Penkower 2010: 2) ist eine religiös begründete Angst von jüdischen Menschen, die befürchten, dass sie oder ihre Kinder nicht heiraten oder nicht angemessen heiraten werden. Diese Angst sei nicht etwa die Angst vor dem Alleinsein, sondern besteht darin, den Ansprüchen der Gemeinschaft nicht zu genügen. Diese verlangt nach „[...] offspring who will provide a link in the chain of Jewish survival'“ (Levitz; zit. nach Penkower 2010: 2). Das Gebot zur endogamen Heirat unterstreicht die Relevanz von Verwandtschaft und der damit einhergehenden Reproduktion der jüdischen Gemeinschaft: „Marriage is thus elevated from personal pursuit to one with the existential weight of an entire nation behind it" (Penkower 2010: 3).

Religiöse Endogamie ist massgebliches Mittel, um den Staat Israel als jüdische Nation zu erhalten (vgl. Karayanni 2010). Die matrilineare Weitergabe der Religion wird auf nationaler Ebene durch die automatische Staatsbürgerschaft, die der jüdischen Gemeinschaft in Israel gesetzlich gewährt wird, getragen. Bereits mit Israels Unabhängigkeitserklärung vom 14. Mai 1948 wurde dieses Recht verbrieft. Zusätzlich garantiert das Rückkehrgesetz automatische israelische Staatsbürgerschaft für Mitglieder der jüdischen Gemeinschaft, so auch den Diaspora-Gemeinschaften. Das Rückkehrgesetz „erlaubt allen Personen, die ihre jüdische Abstammung bis zu einem jüdischen Grosselternteil zurückverfolgen können, sowie ihren Ehepartnern, nach Israel einzuwandern" (Averbukh und Kranz 2016: 3). Das israelische Staatsbürgerschaftssystem beruht demnach nicht auf einem Rechtsrahmen „der Zugang zur Staatsbürgerschaft [...] individuell, sondern nach Gruppenzugehörigkeit bestimmt“ (Averbukh und Kranz 2016: 7).

Da jüdische Gemeinden gemäss Goldlust eher klein sind, bieten sich nur wenige Möglichkeiten, eine ${ }^{\star} n$ passende ${ }^{\star} n$ Partner$^{*}$ in zu finden. Durch neu kreierte Verbindungen und Heiratsbeziehungen zwischen verschiedenen Regionen entstand jedoch ein vergrössertes 
Netzwerk, wobei die einzelnen Gemeinschaften zu translokalen ethno-religiösen Vergemeinschaftungen wachsen konnten. Der Angst, dass die jüdische Gemeinschaft durch Assimilation oder Adaption von Gruppen kontinuierlich in eine "mainstream-society“ (Goldlust 2001: 17) verschwindet, wird mit einer Verstärkung der In-Group-Solidarität entgegengewirkt. Reproduktion wird zum gemeinsamen Interesse und ist nicht mehr nur rein biologisch begründet. Auch Annette Weiner (1978) beschreibt Reproduktion als nicht ausschliesslich biologischen, sondern kulturellen Prozess. Sarah Franklin (1997) spezifiziert Weiners Aussage und ergänzt, dass Biologie zwar ein Lebewesen erzeuge, nicht aber Mitglieder einer Gemeinschaft. Das Fortbestehen der Gesellschaft wird somit durch die Weitergabe der kulturellen Identität gesichert. In diesem Zusammenhang gewinnt auch die Heirat, der im traditionellen Sinn erste Schritt in der Familienplanung, besondere Bedeutung.

Um die Reproduktion der Nation und damit einhergehende Sicherung des Fortbestands des Judentums vertieft zu thematisieren, sind zwei Konzepte von Don Handelman (1994) hilfreich: le'om (Nationalität) und edot (Ethnizität).

Handelmans Konzept des le'om, was mit Nationalität übersetzt werden kann, schafft einen Prozess, der eine Gleichheit nur innerhalb der jüdischen Gemeinschaft und eine Ungleichheit zwischen Juden* und Nicht-Juden* schafft. Durch die Wichtigkeit und Stärkung der jüdischethnischen Identität entsteht, verknüpft mit der Nationalität, ein starker Zusammenhalt. Dieses Konzept der Abgrenzung gegen aussen schliesst die in der Diaspora lebende jüdische Gemeinschaft als Teil eines grossen Ganzen mit ein.

Das Konzept des edot betont die Einheit innerhalb des jüdischen Kollektivs im Sinne kultureller Gleichheit innerhalb jüdischer Gemeinschaften in der Diaspora. Handelman erklärt das Konzept historisch anhand eines Kuchens, der durch die Vertreibung ins Exil und in DiasporaGemeinschaften in einzelne Stücke, jüdische Subkulturen, geteilt wird. Mit der Errichtung des modernen Staates, kehrten diese Stücke an ihren territorialen Ursprung zurück. Heute ist die Sichtweise dieselbe: „These partly divided slices - all now within the unbroken circle of peoplehood and statehood - are the edot of Jewish Israel" (Handelman 1994: 451). Edot betont so die Einheit und Gleichheit innerhalb einer gut definierten und klar abgegrenzten jüdischen Gemeinschaft.

Beide Konzepte, le'om und edot, kreieren einen Diskurs der Ungleichheit und der Hierarchisierung zwischen jüdischer und nicht-jüdischer Gemeinschaft in Israel, wie auch ein Gefühl von Zugehörigkeit in den Diasporas aufgrund einer wesentlichen Gleichheit im Innern. „In sum, 'nationality' provides the epistemological grounds for ethnic equality among Jews; while the idea of a Jewish ethnicity promotes inequality between Jew and non-Jew" (Handelman 1994: 443). Folglich ist das Heiraten innerhalb des Judentums nicht nur von persönlichem, sondern auch von nationalem Interesse:

From the perspective of diasporic minority, the combination of powerful group boundary maintenance mechanisms and strong motivating ideologies, from without and/or within, is more likely to ensure group continuity. A mutual construction of a strong sense of social distance and 'otherness' from the 'host society', invariably based on one or more perceived differences of race, ethnicity, religion, culture or language, is usually quite effective. (Goldlust 2001: 14) 


\section{Jüdische Heiratsvermittler*innen in der Praxis}

Die Partner*innensuche empfinden viele Menschen als eine existenzielle Herausforderung. Auch für die jüdisch-orthodoxe Gemeinschaft, die Ariel Penkower (2010) untersucht hat, trifft das zu. In den letzten Jahren, so Penkower, steigt die Zahl alternder und alleinstehender jüdisch-orthodoxer Personen, die nach Ehepartnern suchen. Als Lösungsstrategien gelten unter anderem professionelle Partnervermittlungen oder Onlinedating-Plattformen. Im folgenden Teil soll die Online-Partnervermittlung Simantov International vorgestellt werden, um später eine Synthese zwischen theoretischen Konzepten und den thematisch relevanten Aspekten anzustellen.

\section{Schadchen unterstützen den Schidduch}

Heiratsvermittler*innen geniessen eine lange Tradition in der jüdischen Gesellschaft, vor allem in Europa. Nach Peres et al. (1980) besteht ihre traditionelle Rolle darin, auf Anfragen von Müttern mit Töchtern im Heiratsalter einen passenden Ehemann zu finden. Danach ist ihre Aufgabe jedoch noch nicht beendet. Heiratsvermittler*innen, Eheanbahner*innen oder Schadchen nehmen im Weiteren eine vermittelnde und schlichtende Rolle während den prämaritalen Verhandlungen und Recherchen, dem sogenannten Schidduch, ein. Schidduch steht für den gesamten Prozess vom Vorstellen über intensive Personenrecherche bis zum Verhandeln des Mitgifts. Erst wenn sich die beiden Familien geeinigt haben, werden sie für ihre Arbeit vergütet.

José Weber ist einer der erfolgreichsten jüdischen Heiratsvermittler, Mitbegründer der Heiratsvermittlung Simantov International und sieht sich in seiner Rolle als Shaliach, „ein Abgesandter Gottes auf Erden“ (Knüppel 2013: o. S.). Seine Aufgabe versteht er darin, für die in der Diaspora lebenden Jüdinnen und Juden ihren Beshert, ihren „Seelenverwandten“, zu finden: „Zufälle gibt es nicht im Judentum, denn Gott lenkt alle Geschehnisse“ (Schenkel 2018: o. S.).

Anders als herkömmliche Online Dating-Plattformen verspricht Simantov International, Paare zusammenzubringen, die auch wirklich zusammengehören. Denn für jüdische Singles in nichtjüdischen Mehrheitsgesellschaften ist es schwierig, den passenden Partner zu finden. „Wir verlangen den Nachweis einer Mitgliedschaft in einer jüdischen Gemeinschaft oder eine Bestätigung vom Rabbiner, um sicherzugehen, dass es sich wirklich um einen jüdischen Menschen handelt" (José Weber; zit. nach Zehden 2017: 36).

Wenn sich ein toller Katholik bei mir meldet, muss ich ihn also enttäuschen. Ich bin nicht übermässig religiös, aber es ist meine Überzeugung, dass vor allem junge Menschen, die irgendwann Kinder haben werden, der gleichen Glaubensrichtung angehören sollten. Alles andere gibt nach der ersten Verliebtheit nur Probleme. (José Weber; zit. nach Müller 2012: 11)

Dieser Ausschnitt aus einem Interview zeigt auf, dass für den Heiratsvermittler José Weber interreligiöse Ehen ein Herd für familiäre Probleme sind. Um den jeweiligen guten Match für eine Person zu finden, wendet er seine eigene Befragungstaktik an. Nebst der Online-Registrierung auf der offiziellen Internetseite von Simantov International, dabei wird vor allem der Grad an 
Religiosität überprüft, werden die Kandidatinnen und Kandidaten beim ersten Skype-Telefonat einer kurzen, aber wichtigen Befragung zu ihren jüdischen Wurzeln unterzogen:

Ich frage dann zum Beispiel: ,Woher kommt deine Omi, woher dein Opi? Was ist eine Barmizwa, und was bedeutet die Zahl Sieben im Judentum?' Ein richtiger Jude wisse, dass man mit der Barmizwa die religiöse Mündigkeit erlange und die Sieben eine Sonderstellung in der jüdischen Religion einnehme. (José Weber; zit. nach Makowski 2014: 39)

Das traditionelle Vorgehen legt demnach grossen Wert darauf, Partner ${ }^{\star}$ innen zu finden, die eine stabile familiäre Einheit bilden (vgl. Milevsky, Shifra Niman, Raab und Gross 2011). „Wir wollen ja eigentlich, dass man ineinander verliebt ist, das ist kein Tabuthema" (Bondolfi 2013: o. S.), erklärt eine jüdisch-orthodoxe Zürcherin in einem Interview mit der Neuen Zürcher Zeitung. „Es sei einfach schwieriger, sich zu verlieben, weil die Geschlechter viel getrennter lebten“ (Bondolfi 2013: o. S.). Heiratsvermittler*innen werden in jüdisch-orthodoxen Gemeinden eingesetzt, da „bereits ab der ersten Grundschulklasse [...] die meisten orthodoxen Kinder nach Geschlechtern getrennte Schulen [besuchen]“ (Bondolfi 2013: o. S.). Sogenannte Mischehen sind gemäss der Halacha, dem Religionsgesetz, nicht erlaubt.

Besonders problematisch sind aus orthodoxer Sicht Ehen zwischen einem jüdischen Mann und einer nichtjüdischen Frau, da in diesem Fall die Kinder nicht jüdisch sind. Nach dem Religionsgesetz kommt eine solche Mischehe dem Tod des jüdischen Sohnes gleich. (Bondolfi 2013: o. S.)

In der Praxis der Heiratsvermittler ${ }^{\star}$ innen verschwindet scheinbar der Unterschied zwischen persönlichem Glück und der gesellschaftlichen Reproduktion.

Obwohl Weber nach eigenen Angaben selber nicht praktizierend ist, spielt die jüdische Tradition eine zentrale Rolle.

In der jüdischen Tradition ist es sehr wichtig, zu heiraten und Kinder zu bekommen. [...] Schon in der Tora stehe: Hast du keine Kinder, lebst du nicht. [...] Den Ultraorthodoxen [sei er jedoch] nicht koscher genug. (José Weber; zit. nach Makowski 2014: 39)

Im Interview mit der Jüdischen Rundschau erklärt er, dass trotzdem genau diese Sorgfalt Simantov International von herkömmlichen Onlinedating-Portalen unterscheide (vgl. Zehden 2017).

Ausserdem liegt der Partner*innensuche kein Algorithmus zugrunde, sondern Weber erstellt während den Gesprächen mit den Suchenden ein Profil, das er dann mit bereits erfassten Profilen seiner Kartei abgleicht und nach jedem nicht erfolgreichen Treffen spezifiziert. Eine romantische Bindung an eine ${ }^{\star} n$ Partner ${ }^{*}$ in vor der Heirat wird in der traditionellen jüdischen Gesellschaft nicht gerne gesehen. Ultraorthodoxe Jüdinnen beschreiben den Sinn und Zweck des Datings allein im Herausfinden des „geeigneten“ Partners und nicht als Vergnügen (vgl. 
Milevsky et al. 2011). Weber wählt selber die infrage kommenden Partner*innen aus und gibt Tipps für ein gelungenes Kennenlernen über Skype.

Dank Vernetzung durch das Internet entstehen translokale Verbindungen jüdischer Gruppen in der Diaspora, denen Identität und Nationalität wichtig genug ist, diese auch weiterzugeben (vgl. Goldlust 2001). Mit Hilfe der Heiratsvermittler*innen bleiben jüdische Singles in der Diaspora nicht mehr nur virtuell vernetzt, sondern werden aktiv ermutigt zu heiraten. Ein Beispiel sind Chaim und Ilana Lipschitz, ein jüdisch-orthodoxes Ehepaar aus Zürich, die sich ebenfalls traditionell mittels Heiratsvermittlerin kennenlernten. In ihrem Fall war es jedoch nicht die professionelle Hilfe eines Schadchen wie José Weber, sondern eine Bekannte beider Familien, die nach „göttlichem Plan“ die beiden im Schidduch (Matchmaking-Prozess) einander vermittelte (Schenkel 2018: o. S.). In einem Interview mit der Neuen Zürcher Zeitung beschreibt das Paar die Schwierigkeiten jüdisch-orthodoxer Frauen und Männer, in der eher kleinen jüdischen Gemeinschaft in Zürich oder der Schweiz, eine ${ }^{\star} n$ geeignete ${ }^{\star} n$ Ehepartner ${ }^{\star}$ in zu finden. Sich mittels traditionellem Schidduch, welcher einen gründlichen Rechercheprozess des jeweiligen Gegenübers beinhaltet, von Drittpersonen vermitteln zu lassen, sei so auch ein rationaler Entscheid, da selbst Verheiratete aus der Verwandtschaft oder professionelle Vermittler*innen wissen, worauf es in einer Ehe ankomme. Eine andere Familie aus Zürich betont, dass es darum gehe, Traditionen zu bewahren: „'Das ist genau der Grund, warum wir uns - extrem gesagt abkapseln von der normalen Gesellschaft'. Damit solche Mischehen nicht passieren, denn nur auf diese Weise habe das Judentum überleben können“ (Bondolfi 2013: o. S.).

\section{Netzwerktheoretische Überlegungen}

Ausgehend von der Prämisse, dass alle Menschen mit anderen Menschen in irgendeiner Form interagieren (vgl. Elias 2018), sind für den hiesigen Untersuchungsgegenstand vor allem Theorien der relationalen Soziologie und der sozialen Netzwerke relevant (vgl. Emirbayer 1997). Menschen zeichnen sich gemäss Georg Simmel durch ihre individuellen Positionen in unterschiedlichen sozialen Kreisen aus. Um die Handlungsfähigkeit des einzelnen Menschen besser zu verstehen, eignet sich vorab die Mikroebene als Ausgangspunkt. Gesellschaftliche Strukturen bis hin zur Entstehung von Staaten und Nationen auf der Makroebene hängen mit Mikrophänomenen auf individueller Ebene zusammen und können nicht davon losgelöst betrachtet werden. So werden nachfolgend ebenfalls makrostrukturelle Theorien vorgestellt. Bei der Verbindung von Makro- und Mikroebene

kann die Netzwerkforschung ihr ganzes Potential entfalten; denn sie setzt bei der Mesoebene an, indem sie Figurationen, interdependente Sozialzusammenhänge, Netzwerkformationen zum Gegenstand ihrer Analyse und theoretischen Reflexion erklärt. (Stegbauer und Häussling 2010: 59)

\section{Kreuzung sozialer Kreise und Figurationen}

„Individuen sind für die Soziologie vornehmlich deshalb von Interesse, weil sie einerseits aktiv Wechselwirkungen konstituieren, andererseits aber auch von diesen Wirkungen betroffen 
sind“ (Nollert 2010: 157). Während primäre „soziale Kreise“ natürliche, intime Formen der Vergesellschaftung sind, in die Menschen hineingeboren werden und mittels derer sich „die Mitglieder an gemeinsamen Werten und Normen orientieren“, gibt es aufgrund „zunehmender sozialer Ausdifferenzierung [...] weitere Kreise, die nicht auf Askription, sondern auf gemeinsamen kulturellen, wirtschaftlichen oder politischen Interessen beruhen“ (Nollert 2010: 157). Entscheidet demnach ein Mensch, sich vom ursprünglichen Netzwerk zu lösen, um weitere Kontakte zu knüpfen, so ergibt sich eine neue Konstellation von Zugehörigkeiten zu unterschiedlichen Kreisen, was schliesslich die Individualität eines Menschen ausmacht. Individuen bilden Knoten in einem System sozialer Kreise und erhalten eine multiple Identität.

Nebst der Individualität des Menschen ist auf einer Makroebene die Kreuzung der sozialen Kreise auch Basis sozialer Kohäsion. Jedoch birgt eine mögliche Entkreuzung Konfliktpotential. So kann die Kreuzung sozialer Kreise „die konfliktive Gliederung der Gesellschaft in eine Vielfalt sich gegenseitig abschottender sozialer Kreise“ (Nollert 2010: 161) verhindern. Definieren Menschen demnach ihre Identität nicht nach der Zugehörigkeit zu einem einzigen sozialen Kreis, lassen sich Konflikte vermeiden, da Kreuzungen sozialer Kreise eine „konfliktreduzierende Wirkung“" (Nollert 2010: 161) aufweisen.

Bei dem eben beschriebenen Konzept handelt es sich um die Transkulturalität (vgl. Welsch 2010), das Simmels Argumentation folgt und die Kreuzung sozialer Kreise und eine Individualisierung der Gesellschaft als Grundvoraussetzung für eine stabile moderne Gesellschaft sieht. „Eine transkulturelle Gesellschaft ist demnach durch eine intensive Vernetzung von sozialen Kreisen charakterisiert" (Nollert 2010: 162). Obwohl es sich bei dieser Überlegung nach Nollert um eine wenig komparativ geprüfte Hypothese handelt, ist sie dennoch hilfreich, um Strukturen und Prozesse in einer Gesellschaft nachzuvollziehen.

Im Unterschied zu transkulturellen Identitäten destabilisieren solitäre Identitäten die Gesellschaftsstruktur (vgl. Sen 2006), was wiederum horizontale Ungleichheiten begünstigt (vgl. Stewart 2008). Damit verknüpft ist das Konzept der Parallelgesellschaft und die „Tendenz immigrierter Minderheiten [...] sich von der sogenannten Mehrheitsgesellschaft abzuschotten“ (Nollert 2010: 162). Multikulturalismus positioniert sich gewissermassen in der Mitte der beiden vorherigen Konzepte und postuliert, dass „es möglich sei, eine Gesellschaft zu kreieren, in der ethnisch und kulturell definierte Gruppen in gegenseitigem Respekt nicht miteinander, sondern nebeneinander existieren können“" (Nollert 2010: 162). Nollert (2010: 162) kommt zu einem für die vorliegende Arbeit zentralen Schluss:

[S]oziale Kreise [neigen] offensichtlich unter spezifischen Bedingungen dazu [...], bewusst auf Kreuzungen zu verzichten [...] und kleine Gruppen [können] in einer Gesellschaft ihre Identität, interne Kohäsion und Strukturen nur dann konservieren [...], wenn sie sich nicht assimilieren bzw. möglichst auf intensive Interaktionen mit grösseren, hegemonialen sozialen Gruppen verzichten.

Nollert (2010: 163) folgert, „dass die soziale Kohäsion moderner Gesellschaften darauf beruht, dass ihre Mitglieder nicht in sozialen Netzwerken mit deutlichen Struktur- und Sinngrenzen verbleiben, sondern sich individualisieren“ und Teil mehrerer Netzwerke werden. „Dieses Strukturmuster ist die Grundlage für die Offenheit moderner, funktional differenzierter Gesellschaften“ (Jansen 2007: 7). Sogar im Zuge der Digitalisierung und Globalisierung lassen sich in 
manchen Gesellschaften Muster erkennen, diese Kreuzungschancen entweder abzubauen oder sie bewusst nicht wahrzunehmen. Die soziale Schliessung eines Netzwerks ist somit auf starke Beziehungen und ein engmaschiges Netzwerk angewiesen, wobei sich eine gruppenbezogene Solidaritätsverpflichtung bildet.

Nach Simmel beinhaltet die moderne Gesellschaft jedoch vor allem Vergemeinschaftung. Richter und Nollert (2014) machen ebenfalls darauf aufmerksam, dass bereits Simmel die verschiedenen Formen der Zugehörigkeit und die verschwommenen Grenzen zwischen Gruppen als Merkmal der modernen Gesellschaft gesehen hat. Hierzu hebt Simmel gemäss Stark die Bildung von Gruppen heraus, die den Vergemeinschaftungsprozess unterstützt hinsichtlich des le'om, des Gefühls zu einem grossen Ganzen zu gehören und sich als Teil einer Nation zu wissen.

Als Weiterentwicklung der These von Simmel postuliert Coser, dass Konflikt dazu dient, „die Identität und die Grenzen von Gesellschaften und Gruppen zu schaffen und zu erhalten. Der Konflikt mit anderen Gruppen trägt zur Schaffung und zur Festigung der Gruppenidentität bei und erhält die Grenzen gegenüber der sozialen Umwelt" (Coser 2009: 43). So nimmt der Konflikt, welcher in sozialen Wechselwirkungen durch die Kreuzung sozialer Kreise entsteht, nicht eine destruktive Form an, sondern eine gruppenfestigende Funktion. Kleine Gruppen können sich demgemäss nur so konservieren, indem „sie sich nicht assimilieren bzw. möglichst auf intensive Interaktionen mit grösseren, hegemonialen sozialen Gruppen verzichten “ (Nollert 2010: 162).

Die thematisierten Kreuzungen sozialer Kreise und individuellen Zusammensetzungen, die der Wechselwirkung zugrunde liegen, machen das Individuum aus und „prägen den Einzelnen je nach eingenommener Rolle in essentieller Weise" (Häussling 2010: 65). Auch die relationale Soziologie fokussiert nicht auf einzelne Akteure oder Strukturen, sondern auf Beziehungen und erkennbare Netzwerkstrukturen.

So deutet auch Norbert Elias die „relativ dauerhaften Zusammenschlüsse von Menschengruppen“ (Häussling 2010: 66) als relational. Figurationen, wie Elias diese Interdependenzgeflechte nennt, spielen eine zentrale Rolle in der Sozialisation des Menschen, die „er als ein ,Hineinwachsen' in Figurationen begreift“ (Häussling 2010: 66-67). Menschen bilden in ihrem Zusammenleben untereinander Figurationen, wobei das erlernte Wissen von Generation zu Generation weitergegeben wird: „Ein heranwachsendes Menschenwesen, das keinen Zugang zu Sprach- und Wissenssymbolen einer bestimmten Menschengruppe erworben hat, bleibt ausserhalb aller menschlicher Figurationen [...]“ (Elias 2018: 115).

Auch Elias' Etablierten-Aussenseiter-Figuration lässt sich für die Beantwortung der Forschungsfrage heranziehen und bietet spannende Einsichten in gesellschaftliche Prozesse. Denn das Verhalten einzelner Menschen ist im Kontext der Figurationen zu verstehen, wobei über verinnerlichte Normen Zwänge entstehen. „In diesen Strukturen äussert sich gesellschaftliche Macht“ (Lenz 2017: 246). Das von Elias und Scotson (2002) entwickelte Modell zeigt auf, wie unabhängige Gruppen mit einem starken Wir-Gefühl auf hohe Standards der Selbstbeherrschung und Selbstkontrolle, sowie gegenseitiger und Selbstkontrolle angewiesen sind „um das Gruppencharisma aufrechtzuerhalten“" (Treibel 2008: 82). Gruppencharisma ist ein überhöht positiv besetztes Wir-Ideal, das Mitgliedern ein Gefühl der Überlegenheit vermittelt. Ganz im 
Sinne von Elias und Scotson (2002) wird dieses Modell als Erklärungsmodell zur Analyse der Beziehung von Juden* und Nicht-Juden* herangezogen.

\section{Soziale Netzwerke}

Die grundlegende Idee der sozialwissenschaftlichen Netzwerkanalyse ist, dass Akteure - wie beispielsweise Personen, Organisationen oder Nationalstaaten - durch soziale Beziehungen miteinander verbunden sind. (Haas und Malang 2010: 89)

Die soziologische Netzwerktheorie schliesst an das Paradigma der relationalen Soziologie an, in dem Beziehungen und die daraus entstehenden Muster und Strukturen voneinander abhängig sind. „Somit verschiebt sich die analytische Perspektive weg von Merkmalen [...] hin zur strukturellen Eingebundenheit der Akteure in soziale Netzwerke, das heisst in Beziehungen zu anderen“" (Haas und Malang 2010: 89). In der Analyse sozialer Netzwerke werden (Meso-)Strukturen mit sozialen Eigenschaften ausgestattet und formal beschrieben. „Ziel ist es, sie für die Erklärung individuellen Handelns heranzuziehen und die Entstehung bzw. Veränderung von Strukturen über individuelles Handeln zu erklären " (Jansen 2003: 13). Es geht also darum, das Verhältnis zwischen Individuum und Gesellschaft zu beleuchten: „Man muss also das Ganze, das Netzwerk, untersuchen, um das Verhalten der Teile, der Netzwerkelemente (meist, aber nicht immer Individuen) verstehen und erklären zu können“ (Jansen 2003: 13).

Die (meso-)strukturelle Handlungstheorie von Ronald Burt dient dazu, die Mikro- und Makroebene zu vereinen. Burt kombiniert in seinem Mikro-Makro-Modell die strukturelle mit der Akteur-bezogenen Komponente: „Interessen und Ressourcen von Akteuren [werden] als von ihrer strukturellen Einbettung abhängig begriffen [während gleichzeitig] den Akteuren aber die Möglichkeit der Rückwirkung auf die Strukturen gegeben“ (Jansen 2003: 18) wird.

Burts Modell beinhaltet folgende vier Grundannahmen:

1. Die Makroebene bildet die Gesellschaft als eine relationale und nach Positionen stratifizierte Sozialstruktur.

2. Die Entwicklung eigener Interessen durch einen Akteur wird durch seine Position in der Sozialstruktur geformt.

3. Die Position in der Sozialstruktur und die bereits durch die Position bestimmten Interessen sind die Constraints für die Handlung.

4. Die Handlungen der zweckorientierten Akteure reproduzieren die soziale Struktur und verändern sie unter Umständen. (Jansen 2003: 19)

Ein wichtiger Teil der Sozialstruktur auf der Mesoebene ist das soziale Kapital. Nebst dem, dass einzelne Akteure soziales Kapital aus ihrem Netzwerk beziehen können, geht es auch darum, dessen Verfügbarkeit strategisch beeinflussen zu können. Der Grundgendanke hinter dem Begriff des Sozialkapitals ist, „dass Investitionen in soziale Beziehungen einen Nutzen erwarten lassen“ (Hennig 2010: 177). Gemäss Nan Lin (2001) beeinflussen Ressourcen aus sozialen Netzwerken die Ergebnisse des sozialen Handelns massgeblich. So können soziale Beziehungen „eine Art soziale Empfehlung für ein Individuum sein“ (Hennig 2010: 177). 
Die Zugehörigkeit zu einem Netzwerk wird demnach zu einer wichtigen Ressource, wobei im Fall der endogamen Heiratspraxis der jüdischen Gemeinschaft ein geschlossenes System mit starken Beziehungen entsteht. Dieses Phänomen, dass eine Beziehung oft mit ähnlichen oder bereits bekannten Netzwerkpartner*innen eingegangen wird, wird Homophilie genannt. „Die Wahl bekannter Partner reduziert Unsicherheit und führt zu tendenziell cliquenhaften Netzwerkstrukturen“ (Jansen 2007: 15), was je nach Kontext als positive oder negative Folge der sozialen Schliessung interpretiert werden kann.

Die „gruppenbezogene partikularistische Solidarität und Zugehörigkeit“ (Jansen 2003: 28), die ebenfalls auf sozialen Schliessungsprozessen beruht, ist eine spezifische Form des sozialen Kapitals, auch genannt Strong Ties, „, die starken und häufigen Beziehungen mit hoher Überlappung und Reziprozität in kohäsiven, abgegrenzten Gruppen“ (Jansen 2003: 28). Diese Form der sozialen Schliessung hat neben der bereits angesprochenen positiven Seite eine Kehrseite der verstärkten sozialen Kontrolle für einzelne Individuen.

Im Fall der in der Diaspora lebenden jüdischen Gemeinschaft sind jedoch ebenfalls Brokerpositionen relevant. Neben den kohäsiven Teilen der jüdischen Gemeinschaft, die mit starken Beziehungen zusammengehalten werden, kann die geografische Distanz zwischen den Gemeinschaften strukturelle Löcher erzeugen. Die strukturelle Einbettung der Akteure in ein Netzwerk beeinflusst massgeblich deren individuelle Handlungsfähigkeit. „Eine Person, die mit Personen Verbindungen unterhält, die ihrerseits untereinander nicht direkt verbunden sind, erlangt die Möglichkeit, zwischen diesen Kontakten zu vermitteln und daraus Vorteile zu ziehen“ (Scheidegger 2010: 145). Diese Verbindungen, oder Ties, überbrücken folglich strukturelle Löcher. Gemäss Mark Granovetter (1973) sind Weak Ties aufgrund ihrer Eigenschaften „intransitiv und transzendieren als Brückenbeziehungen das engere Umfeld, womit sie durch Kontakte zu Aussenstehenden die Anbindung an ein grösseres Netzwerk ermöglichen“ (Scheidegger 2010: 145).

\section{Diaspora und digitale Medien}

Im Hinblick auf die Vergemeinschaftungs- und Reproduktionsleistungen jüdischer Heiratsvermittler*innen ist die identifikatorische Definition von Diaspora nach James Clifford (1994) sinnvoll: Diaspora ist „eine hybride, imaginierte, translokale Identität. Diaspora gilt [...] als ein Gefühlszustand, als Selbstverortung in einer immer unübersichtlicheren Welt" (Moosmüller 2002: 11). Die wesentliche Eigenschaft der Diaspora ist, dass sie, im Unterschied zur multikulturellen Gesellschaft, stets eine enge Beziehung mit den Herkunftsländern pflegen und sich diesen noch immer loyal gegenüber verhalten (vgl. Moosmüller 2002).

Die neuen Möglichkeiten, die digitale Medien mit sich bringen, sind auch für DiasporaGesellschaften von Interesse. Medien können einen Beitrag zur "gesellschaftlichen Integration von ,ethnischen Minderheiten’ in Nationalkulturen bzw. Nationalstaaten leisten“ (Hepp 2009: 33). Hepp kategorisiert digitale Medien als ,alle Formen einer im erweiterten Sinne zu verstehenden computervermittelten Netzwerkkommunikation, wozu neben WWW, E-Mail, Social Software/Web 2.0 und anderen Kommunikationsformen des Internets auch das Mobiltelefon zählt“ (Hepp 2009: 33). Die Vernetzung, die digitale Medien ermöglichen, bietet vor allem für Diaspora-Gemeinschaften grosses Potential. 
Durch neue Formen der Kommunikation, sind Netzwerke nicht mehr beschränkt durch die Notwendigkeit der „physischen Kopräsenz“ (Hepp 2009: 34). Forschungszweige, die sich mit der Globalisierung, der Medienkommunikation und transkultureller Kommunikation beschäftigen, postulieren, dass eine „Intensivierung von internen Kommunikationsprozessen“ (Hepp 2009: 36) die Vergemeinschaftung der Diaspora potentiell stabilisiert und sie so als eine Form deterritorialer Vergemeinschaftung zu verstehen ist. „Translokale Vergemeinschaftungen fügen sich damit gerade zunehmend nicht mehr äusserlich in territoriale Bezüglichkeiten (Nation, Nationenbund, Region etc.), sondern in deterritoriale Zusammenhänge, die neben diese getreten sind“ (Hepp 2009: 37). Eine deterritoriale Vergemeinschaftung lässt sich so „als Netzwerk subjektiv gefühlter Zusammengehörigkeit über verschiedene Territorien hinweg“ (Hepp 2009: 38) fassen.

Ausserdem von Interesse, vor allem im Kontext der transnationalen Vergemeinschaftung, ist die Unterscheidung zwischen ways of being und ways of belonging von Peggy Levitt und Nina Glick Schiller, auf die auch Richter und Nollert (2014) verweisen:

Ways of being refers to the actual social relations and practices that individuals engage in rather than to the identities associated with their actions. [...] In contrast, ways of belonging refers to practices that signal or enact an identity which demonstrates a conscious connection to a particular group. (Levitt und Schiller 2004: 1010)

Menschen, die in transnationalen Kontexten leben, kombinieren demnach diese beiden Formen je nach Situation. Transnationalität soll hierbei jedoch von Transkulturalität unterschieden werden.

Während transnationale Vergesellschaftung einen sense of belonging schafft, steht Transkulturalität für die Verbundenheit über verschiedene Kulturen hinweg. „Transculturalism refers to the symmetric merging of elements from different cultures" (Richter und Nollert 2014: 461). Richter und Nollert (2014) kommen zum Schluss, das transnationale Netzwerke und transkulturelle Zugehörigkeit sich immer gemeinsam entwickeln und gegenseitig beeinflussen.

\section{Jüdische Heiratsvermittlung als Gegenstand der Netzwerkforschung}

In diesem Abschnitt wird die Vergemeinschaftungs- und Reproduktionsleistung jüdischer Heiratsvermittler*innen aus der Perspektive der Netzwerkforschung analysiert. Auf der Mikroebene befindet sich das Individuum. Auf der Makroebene bilden gesellschaftliche Strukturen und Prozesse den Staat und die Nation. Diese Einheiten sind interdependent und beeinflussen sich gegenseitig. Jüdische Heiratsvermittlerinnen sind in der Mitte dieses Gefüges zu platzieren; sie agieren als Broker zwischen Mikro- und Makroebene und zwischen Individuum und Nation. Dies soll im Folgenden im Detail diskutiert werden.

Im ersten Teil der Diskussion wird vorwiegend die Position der Heiratsvermittler*innen auf der Mikroebene und deren Auswirkungen auf der Makroebene thematisiert. Im zweiten Teil der Diskussion sollen Aspekte der Makroebene behandelt und im thematischen Kontext eingeordnet werden. Die Praktiken und Aspekte sind jedoch analytisch nicht trennscharf und klar 
einer Ebene zuteilbar. Dennoch eignet sich das Modell, das Fallbeispiel der Vergemeinschaftungs- und Reproduktionsleistungen jüdischer Heiratsvermittler ${ }^{\star}$ innen zu analysieren.

\section{Heiratsvermittler*innen als Beziehungsbroker}

Schadchen wie José Weber übernehmen die Aufgabe des Individuums, eine ${ }^{\star} n$ geeignete $^{\star} n$ Partner*in zu finden, um eine Familie zu gründen und somit einen Beitrag zum Fortbestand der jüdischen Gemeinschaft zu leisten. Sie sind Beziehungsbroker und bilden die Brücke zwischen den in der Diaspora lebenden Jüdinnen und Juden und überwinden so strukturelle Löcher. Durch ihre Vernetzung sind sie in der Lage, sonst sehr geschlossene Netzwerke miteinander zu verbinden. Dennoch ist es für die Reproduktion der Nation (le'om) nötig, das endogame Heiratsregime einzuhalten, wozu Heiratsvermittler*innen beitragen. So profitiert die jüdische Gemeinschaft von der Vernetzung der Heiratsvermittlerinnen, da Homophilie zwar erwünscht ist, aber nicht genügend potentielle Heiratspartner ${ }^{\star}$ innen im eigenen Netzwerk vorhanden sind. Wie von Nollert (2010) beschrieben, kann die jüdische Gemeinschaft als Beispiel betrachtet werden, die bewusst auf die Kreuzung sozialer Kreise verzichten, um ihre Identität und ihre Gesellschaft zu konservieren. Traditionell lebende Jüdinnen und Juden können dank der Vernetzungsarbeit der Heiratsvermittler*innen abgeschottet in einer Parallelgesellschaft leben.

Berücksichtigt man in diesem möglichen Verhalten den Kontext und damit die Figurationen und Interdependenzgeflechte, fällt auf, wie stark die gesellschaftliche Macht und die Kontrolle sind. Denn das Verhalten jeder heiratswilligen jüdischen Person wird durch die Figuration, in der sie aufwächst, mittels Sozialisation erschaffen und gestärkt. Gesellschaftliche Strukturen und deren Wirkung beeinflussen demnach auch die individuelle Handlungsfähigkeit. Jüdische Heiratsvermittler*innen übernehmen so eine weitere Kontrollfunktion zwischen dem israelischen Staat (Staatsbürgerschaft basierend auf jüdischer Gruppenzugehörigkeit), der Nation (jüdischen Gemeinschaft le'om) und dem Individuum. Sie helfen dabei, die jüdische Gemeinschaft vorstellungsgetreu zu reproduzieren, was wiederum die Daseinsberechtigung des Staates Israel bildet. Matchmaking produziert so Mechanismen, die helfen, die strikten gesellschaftlichen Normen in der Partnersuche einzubringen (vgl. Peres et al. 1980). Die Entscheidung, ein ${ }^{\star}$ e Heiratsvermittler*in zu engagieren, um das endogame Heiratsregime durchzusetzen, zeigt die starke Einbettung der jüdischen Gemeinschaft in gesellschaftliche und religiöse Traditionen, was wiederum ein verstärktes Wir-Gefühl fördert und die otherness zur nicht-jüdischen Gesellschaft untermauert (edot).

Diese Einbettung in den Netzwerkkontext liefert, wie von Burt beschrieben, eine Erklärung für das individuelle Handeln und verbindet analytisch die Mikro- mit der Makroebene. In Burts Modell bildet die gesellschaftliche Struktur die Makroebene. Im Fallbeispiel steht dies für das religiös-endogame Heiratsregime, welches zwingend eingehalten werden muss, um die jüdische Gemeinschaft, welche matrilinear weitergegeben wird, zu reproduzieren. Die Reproduktion der jüdischen Nation hängt davon ab. Denn wenn die Kontinuität des Judentums gesichert wird, so wird auch der Staat Israel aufgrund der angesprochenen Rechtslage und Gesetze aufrechterhalten. So werden im Rahmen des Nationalismus die Interessen der Akteure, welche in der gesellschaftlichen Struktur positioniert und in soziale Kreise eingebettet sind, über die Zeit hinweg geformt. Jüdinnen und Juden, die in einer traditionellen Familie aufgewachsen sind, werden durch die Beziehungen und Figurationen auf eine entsprechende Weise sozialisiert. Sie 
übernehmen Vorstellungen und implementieren diese in ihr eigenes Leben gemäss traditionellreligiöser gesellschaftlicher Normen und Werte. Constraints, also Einschränkungen für das individuelle Handeln, sind gemäss Burt sowohl die Position in der Sozialstruktur als auch „die bereits durch die Position bestimmten Interessen“ (Jansen 2003: 19).

Wenn eine im traditionellen, orthodoxen Milieu aufgewachsene jüdische Person das religiös-endogame Heiratsregime nicht befolgt oder kein Interesse daran zeigt, die jüdische Kontinuität zu sichern, kann die Person soziale Konsequenzen spüren oder gar aus der Gruppe ausgeschlossen werden, wie folgende Aussage eines jüdisch-orthodoxen Paares aufzeigt: „In die Synagoge dürfe jeder kommen [...] 'Es wird niemand angesprochen: He! Du hast eine nichtjüdische Frau, du gehörst nicht mehr zu uns!' [...] 'Aber er weiss, was wir denken'“ (Bondolfi 2013: o. S.). Die Zugehörigkeit zu einem Netzwerk ist also eine wichtige Ressource. Das jüdische Diaspora-Netzwerk basiert auf einer gruppenbezogenen partikularistischen Zugehörigkeit, was zu sozialen Schliessungs- und Abschottungsprozessen führt. Die Solidarität, die in solchen Gesellschaftsstrukturen stattfindet, führt zur verstärkten sozialen Kontrolle, was wiederum individuelles Handeln einschränkt und in eine gewünschte Richtung lenkt. Die vom Individuum letztlich gewählte Handlung schliesst den Kreis insofern, als dass sie ihrerseits die soziale Struktur reproduziert.

Wie bereits im theoretischen Rahmen aufgezeigt, prägt das Sozialkapital die Sozialstruktur. Und die darin entwickelten sozialen Beziehungen bringen einen Nutzen, indem sie soziales Handeln beeinflussen. Im Fallbeispiel ist das soziale Kapital vor allem für die Heiratsvermittler*innen, seien sie professionell wie José Weber oder Bekannte, die den Schidduch unterstützen, eine essenzielle Ressource, da es zu erfolgreichen Paarungen beitragen kann. Besonders bei professionellen Beziehungsbrokern ist die Position zwischen den einzelnen Diaspora-Netzwerken strategisch wichtig für die Reproduktion der Nation. Einziger Unterschied zum vorgestellten Modell von Burt ist, dass Heiratsvermittler*innen in ihrer Tätigkeit keine Strong Ties zu einem Cluster führen, sondern zentral positioniert sind, mit wichtigen Weak Ties.

\section{Heiratsvermittler*innen und der jüdische Nationalstaat}

Auch Strukturen auf Makroebene beeinflussen die Heiratsvermittlung. Benedict Anderson definiert die jüdische Gemeinschaft als

imagined community: It is imagined because the members of even the smallest nation will never know most of their fellow-members, meet them, or even hear of them, yet in the minds of each lives the image of their communion. (Anderson 2006: 6)

Die jüdische Gemeinschaft ist mitunter ein Diaspora-Netzwerk, da ihre Identität translokal definiert wird und ein starkes Wir-Gefühl bei den Mitgliedern herrscht. Drei Merkmale von Diasporas sollen nun im Kontext des Fallbeispiels aufgegriffen werden:

„Institutionen und Netzwerke existieren, die die soziale Kohäsion herstellen und eine gewisse Unabhängigkeit von der Residenzgesellschaft ermöglichen“ (Moosmüller 2002: 13). Das für orthodoxe Juden ${ }^{\star}$ geltende endogame Heiratsregime und die traditionellen Werte und Normen wie die vorgestellten Konzepte der Nationalität (le'om) und der Ethnizität (edot) sichern die soziale Kohäsion. Die Zugehörigkeit zu einem sozialen Netzwerk ist ausserdem eine 
wichtige Ressource, wobei in einer Gemeinschaft, die sich gegen aussen distanziert und sich wie die jüdische Gemeinschaft teils auch abschottet, die Zugehörigkeit sogar noch wichtiger werden kann. Dank dem in Israel geltenden Rückkehrgesetz sind die Diaspora-Gemeinschaften unabhängig von der Residenzgesellschaft.

„Eine Elite existiert, die ein besonderes Interesse an der Aufrechterhaltung der DiasporaGemeinschaft hat" (Moosmüller 2002: 13). Auch hier kann auf die Politik und die Gesetzgebung Israels verwiesen werden. Für die translokalen ethno-religiösen Communities, die Diasporas, ist es wichtig vernetzt zu bleiben. Dies gelingt mittels Einhaltung des endogamen Heiratsregime und der Unterstreichung der Wichtigkeit der Familiengründung, um die Kontinuität des Judentums zu sichern. Die Reproduktion wird so zum kulturellen Prozess, an dem die gesamte Gesellschaft interessiert ist.

„Erwartet wird, gegenüber anderen Diasporas (der eigenen Ethnie), auch über Ländergrenzen hinweg, solidarisch zu handeln (was zur Bildung transnationaler Netzwerke führt)“ (Moosmüller 2002: 13). Die Parallelgesellschaft der traditionellen jüdischen Diaspora bleibt unter anderem dank der Arbeit von Heiratsvermittler*innen vernetzt und kann sich so reproduzieren. Auch der gesetzliche Rahmen ermöglicht, Teil einer Gemeinschaft zu sein, trotz Fehlens einer territorialen Verbindung.

Dank digitalen Medien wird dieser Punkt vereinfacht. „For Jews who are positively inclined towards maintaining a strong Jewish self-identity and concerned with securing Jewish continuity in the future, the Internet is seen as a valuable tool to be embraced" (Goldlust 2001: 26). Denn digitale Medien ermöglichen eine deterritoriale Vergemeinschaftung, eine rein subjektiv gefühlte Zusammengehörigkeit über das Territorium und die physische Kopräsenz hinweg. The Web 2.0] „has the potential, for Jews at least, in reinforcing the cross-national basis of Jewish identity and more effectively maintaining and expanding connections beyond local" (Goldlust 2001: 26).

Hepps Modell der translokalen Vergemeinschaftung zeigt, dass eine territoriale Gesellschaft regional verbunden ist und sich ebenfalls zu einer Nation zusammenschliessen kann. Zur deterritorialen Vergemeinschaftung hingegen gehören Diaspora-Netzwerke und religiöse Vergemeinschaftungen. Beide Aspekte sind für das Fallbeispiel wichtig, da Israel ein jüdischer Nationalstaat ist und per Gesetzgebung allen gebürtigen Juden* die Staatsbürgerschaft verleiht. Schliesslich sind Israel und das Judentum folgendermassen verbunden: Israel als Staat ist zwar territorial gebunden. Doch da Israel ein jüdischer Staat ist, stellt er auch eine Nation dar und deterritoriale jüdische Diasporas, die ethno-religiöse Aspekte teilen, gehören zu ebendieser dazu. Israel wird so zum Nationalstaat und die jüdische Gemeinschaft zur imagined community (vgl. Anderson 2006), die u.a. dank digitaler Medien, aufrechterhalten wird.

Die jüdische Heiratsvermittlung zeigt auch auf, wie der existenziellen Angst des traditionellen Judentums zur Zeit der digitalen Medien und des Onlinedatings begegnet wird. Anders als bei beliebigen Dating-Plattformen, erstellen die User ihr Dating-Profil nicht selber, sondern die Heiratsvermittler*innen übernehmen diese Verantwortung. So entziehen sich beliebige DatingPlattformen der sozialen Kontrolle der Gemeinschaft, indem sie nicht verhindern können, dass Benutzer*innen falsche Informationen über sich publizieren. Auch das Matchmaking wird nicht einem Algorithmus oder den Usern überlassen, sondern den Fähigkeiten und Vernetzungsaktivitäten der Heiratsvermittler*innen. Durch beide Vorsichtsmassnahmen sollen nur 
jüdische Paare zusammengeführt werden, die vor allem auch der Gemeinschaft, aber auch des Nationalstaats mittels Reproduktion dienen. Zusätzlich sichern sich die Schadchen eine Machtposition in der traditionellen jüdischen Gemeinschaft, das ihrem finanziellen Eigeninteresse sowie dem gemeinschaftlichen Reproduktionsinteresse dient.

\section{Schlussfolgerung}

Das Ziel des vorliegenden Beitrags war, die Rolle der traditionellen jüdischen Schadchen mittels netzwerktheoretischer Überlegungen genauer zu betrachten und ihre Auswirkungen auf partner*innensuchende Jüdinnen und Juden, auf die jüdische Diaspora, sowie auf das gesamte Judentum und den Staat Israel hin zu beleuchten. Die jüdische Gemeinschaft ist ein Beispiel einer ethno-religiösen translokalen Vergemeinschaftung, die sich demnach auch ausserhalb ihres Nationalstaats Israel in Diaspora-Netzwerken auf der ganzen Welt organisiert. In den traditionellen Gemeinschaften scheinen sie wenig sozial ausdifferenziert, was vermuten lässt, dass die Mitglieder solcher Diaspora-Netzwerke stark von Figurationen und deren Wechselwirkungen beeinflusst werden. Aufrechterhalten wird die Parallelgesellschaft der traditionellen Jüdinnen und Juden mittels bewussten Verzichts auf die Kreuzung sozialer Kreise, da sich nur so die jüdische Nation sichern und reproduzieren lässt. Die Gruppe bildet ein starkes Wir-Gefühl mit Solidaritätsverpflichtung und entsprechender Erwartungshaltung. Damit dies funktioniert, müssen gesellschaftliche Strukturen und Prozesse existieren, die das Verhalten des Individuums entsprechend beeinflussen.

Die Netzwerkforschung geht davon aus, dass Menschen in soziale Strukturen (Makro- und Mesoebene) eingebettet sind, die deren Handeln beeinflussen (vgl. Burt 1982). Die jüdische Heiratsvermittlung dokumentiert, dass die soziale Reproduktion der Nation eine existenzielle Aufgabe bildet und deshalb die Heiratsvermittler*innen als Beziehungsbroker zwischen global verstreuten Netzwerken eine für den Nationalstaat Israel essenzielle Funktion einnehmen.

Die auf der Makroebene herrschende Kultur, die endogame Heirat vorschreibt, schliesst die Gruppe zusätzlich nach aussen ab und stärkt den internen Zusammenhalt. Das Judentum als Religion beeinflusst das individuelle Verhalten jedoch weiter. Da der jüdische Glaube matrilinear vererbt wird, wird Reproduktion zum kulturellen Prozess. Zusammen mit der Gesetzgebung, der zufolge Israel ein jüdischer Staat ist und ein Rückkehrgesetz existiert, spürt vermutlich jede stark traditionell orientierte jüdische Person den existenziellen ethno-religiösen Druck, heiraten und eine Familie gründen zu müssen.

Obwohl das Web 2.0 bei der individuellen Vernetzung und Partner*innensuche unterstützen könnte, so wollen traditionelle Heiratsvermittler*innen den gesamten Prozess eigens steuern und kontrollieren. Schadchen nützen das Potenzial der Onlinedating-Plattformen, um mit Heiratssuchenden Jüdinnen und Juden in Kontakt zu treten und sich so eine Datenbank anzulegen. Das Matchmaking überlassen sie weder den heiratswilligen Usern selber noch einem Algorithmus. Das persönliche Schicksal wird in die Hände Anderer gelegt, die dank ihres sozialen Kapitals grosses Vertrauen geniessen.

Die Vergemeinschaftungs- und Reproduktionsleistung jüdischer Heiratsvermittler*innen zeigten auf, dass eine grosse Interdependenz zwischen Mikro- und Makroebene besteht. Obwohl es sich dabei um ein stark vereinfachtes Modell handelt, das die Praktiken nicht 
trennscharf zuordnen kann, wird klar, dass die Heiratsvermittler*innen eine strategisch wichtige Position innehaben. Sie befinden sich in einem strategischen Dreieck aus Religion (jüdische Gemeinschaft definiert durch le'om), Staat (Israel als Nationalstaat) und der Reproduktionsforderung, deren Auswirkungen auf der Mikro- wie auch auf der Makroebene klar sichtbar werden. Letztlich fördern sie durch ihre Arbeit die soziale Kohäsion und weitere Existenz der traditionellen (orthodoxen) jüdischen Diaspora.

Der netzwerktheoretische Blick, erweitert um Konzepte wie Transkulturalität, Parallelgesellschaft, Multikulturalismus, Sozialkapital und Beziehungsbroker sowie dessen Einbettung in ein Mehrebenen-Modell (Mikro-, Meso- und Makroebene), in dem Sozialstruktur und Handlung im Hinblick auf die Vergemeinschaftungs- und Reproduktionsleistungen verknüpft werden, dürfte sich allerdings auch für die Analyse anderer religiöser Gemeinschaften eignen.

\section{Danksagung}

Die Autorin bedankt sich bei den externen Gutachterinnen und Gutachtern und den Redaktorinnen und Redaktoren des Journals sozialpolitik.ch für die wertvollen Hinweise und Kommentare.

\section{Deklaration von Interessenkonflikten}

Die Autorin deklariert keine Interessenkonflikte in Bezug auf Forschung, Autorinnenschaft und Publikation des Artikels.

\section{Finanzierung}

Die Autorin hat keine finanzielle Unterstützung für die Forschung, die Autorinnenschaft und die Publikation dieses Artikels erhalten.

\section{Literatur}

Anderson, Benedict (2006). Imagined communities. Reflections on the Origin and Spread of Nationalism (Rev. ed). London: Verso.

Averbukh, Lidia und Dani Kranz (2016). Exklusiver Zugang zur israelischen Staatsbürgerschaft. Bewahrung der jüdischen Mehrheit als demographisches Ziel. Stiftung Wissenschaft und Politik (58), $1-8$.

Bondolfi, Sibilla (2013). „Sabbat-Papi“ und Heiratsvermittler. Neue Zürcher Zeitung. Zugriff am 26. April 2020 auf https://www.nzz.ch/zuerich/sabbat-papi-und-heiratsvermittler-1.18146092.

Burt, Ronald (1982). Toward a Structural Theory of Action. Network Models of Social Structure, Perception, and Action. New York etc.: Academic Press.

Clifford, James (1994). Diasporas. Cultural Anthropology 9(3), 302-338. 
Coser, Lewis A. (2009). Theorie sozialer Konflikte (Neuaufl.). Wiesbaden: VS Verlag für Sozialwissenschaften.

Elias, Norbert (2018). Figuration. In: Kopp, Johannes und Anja Steinbach (Hg.). Grundbegriffe der Soziologie. Wiesbaden: Springer Fachmedien Wiesbaden, 115-117.

Elias, Norbert und John L. Scotson (2002). Etablierte und Außenseiter. Frankfurt am Main: Suhrkamp.

Emirbayer, Mustafa (1997). Manifesto for a Relational Sociology. American Journal of Sociology, 103(2), 281-317.

Franklin, Sarah (1997). Embodied Progress. A Cultural Account of Assisted Conception. London: Routledge.

Goldlust, John (2001). Globalizing Community. Jews in Space. Social Analysis: The International Journal of Social and Cultural Practice, 45(1), 12-31.

Granovetter, Mark S. (1973). The Strength of Weak Ties. American Journal of Sociology, 78(6), 13601380.

Haas, Jessica und Thomas Malang (2010). Beziehungen und Kanten. In: Stegbauer, Christian und Roger Häussling (Hg.). Handbuch Netzwerkforschung. Wiesbaden: VS Verlag für Sozialwissenschaften, 8998.

Handelman, Don (1994). Contradictions between Citizenship and Nationality. Their Consequences for Ethnicity and Inequality in Israel. International Journal of Politics, Culture, and Society, 7(3), 441459 .

Häussling, Roger (2010). Relationale Soziologie. In: Stegbauer, Christian und Roger Häussling (Hg.). Handbuch Netzwerkforschung. Wiesbaden: VS Verlag für Sozialwissenschaften, 63-87.

Hennig, Marina (2010). Soziales Kapital und seine Funktionsweise. In: Stegbauer, Christian und Roger Häussling (Hg.). Handbuch Netzwerkforschung. Wiesbaden: VS Verlag für Sozialwissenschaften, 177-189.

Hepp, Andreas (2009). Digitale Medien, Migration und Diaspora. Deterritoriale Vergemeinschaftung jenseits nationaler Integration. In: Hunger, Uwe und Kathrin Kissau (Hg.). Internet und Migration. Theoretische Zugänge und empirische Befunde. Wiesbaden: VS Verlag für Sozialwissenschaften, 3351.

Jansen, Dorothea (2003). Einführung in die Netzwerkanalyse. Grundlagen, Methoden, Anwendungen (2., erw. Aufl.). Opladen: Leske+Budrich.

Jansen, Dorothea (2007). Theoriekonzepte in der Analyse sozialer Netzwerke. Entstehung und Wirkungen, Funktionen und Gestaltung sozialer Einbettung. FÖV Discussion Papers 39, 1-37.

Karayanni, Michael M. (2010). In the Best Interests of the Group. Religious Matching under Israeli Adoption Law. Berkeley Journal of Middle Eastern and Islamic Law (3), 1-80.

Knüppel, Leila (2013). Ein jüdischer Heiratsvermittler in Deutschland. Deutschlandfunk. Zugriff am 14.08.2019 auf https://www.deutschlandfunk.de/judentum-ein-juedischer-heiratsvermittler-indeutschland.886.de.html?dram:article id=270498.

Lenz, René (2017). Norbert Elias und John L. Scotson. Etablierte und Außenseiter. In: Eckardt, Frank (Hg.). Schlüsselwerke der Stadtforschung. Wiesbaden: Springer Fachmedien Wiesbaden, 239-259. 
Levitt, Peggy und Nina Glick Schiller (2004). Conceptualizing Simultaneity. A Transnational Social Field Perspective on Society. International Migration Review, 38(3), 1002-1039.

Lin, Nan (2001). Social Capital. A Theory of Social Structure ans Action. Cambridge: Cambridge University Press.

Makowski, Elisa (2014). Vergiss nicht, Blumen zu kaufen. Frankfurter Allgemeine Zeitung, 04.07.2014, 39.

Milevsky, Avidan, Deborah Shifra Niman, Atara Raab und Ruchie Gross (2011). A Phenomenological Examination of Dating Attitudes in Ultra-Orthodox Jewish Emerging Adult Women. Mental Health, Religion \& Culture, 14(4), 311-322.

Moosmüller, Alois (2002). Diaspora. Zwischen Reproduktion von „Heimat“, Assimilation und transnationaler Identität. In: Moosmüller, Alois (Hg.). Interkulturelle Kommunikation in der Diaspora. Die kulturelle Gestaltung von Lebens- und Arbeitswelten in der Fremde. Münster: Waxmann, 11-28.

Müller, Franziska (2012). Hochzeit. Die Matchmaker. Die Weltwoche, 14.03.2012, 11.

Nollert, Michael (2010). Kreuzung sozialer Kreise. Auswirkungen und Wirkungsgeschichte. In: Stegbauer, Christian und Roger Häussling (Hg.). Handbuch Netzwerkforschung. Wiesbaden: VS Verlag für Sozialwissenschaften, 157-165.

Penkower, Ariel Y (2010). The Culture of Dating and Single Life in the Modern Orthodox Jewish Community. A Qualitative Study. Unveröffentlichte Dissertation. Department of Psychology, Rutgers University-Graduate School of Applied and Professional Psychology, University of New Jersey, New Brunswick.

Peres, Yochanan, Liora Meisels und Ruth Frank (1980). Commercial Matchmaking in Modern Israel. A Case of Dubious Rationality. Journal of Comparative Family Studies, 11(4), 475-483.

Richter, Marina und Michael Nollert (2014). Transnational Networks and Transcultural Belonging. A Study of the Spanish Second Generation in Switzerland. Global Networks, 14(4), 458-476.

Scheidegger, Nicoline (2010). Strukturelle Löcher. In: Stegbauer, Christian und Roger Häussling (Hg.). Handbuch Netzwerkforschung. Wiesbaden: VS Verlag für Sozialwissenschaften, 145-155.

Schenkel, Lena (2018). Verlobt, verheiratet, verliebt: Wie zwei Jüdisch-Orthodoxe in Zürich zwecks Heirat verkuppelt werden. Neue Zürcher Zeitung. Zugriff am 20. Februar 2020 auf https://www.nzz.ch/zuerich/verlobt-verheiratet-verliebt-wie-zwei-juedisch-orthodoxe-zwecks-heirat-verkuppelt-werden-ld.1447525.

Sen, Amartya (2006). Identity and Violence. The Illusion of Destiny. New York, NY: Norton.

Simantov International (2018). Elite Jewish Matchmaking for Extraordinary Singles. Zugriff am 14.08.2019 auf https://simantov-international.com/.

Stegbauer, Christian und Roger Häussling (2010). Einleitung. Selbstverständnis der Netzwerkforschung. In: Stegbauer, Christian und Roger Häussling (Hg.). Handbuch Netzwerkforschung. Wiesbaden: VS Verlag für Sozialwissenschaften, 57-60.

Stewart, Frances (2008). Horizontal Inequalities and Conflict. Understanding Group Violence in Multiethnic Societies. Basingstoke: Palgrave Macmillan.

Treibel, Annette (2008). Figurationen. In: Die Soziologie von Norbert Elias. Eine Einführung in ihre Geschichte, Systematik und Perspektiven. Wiesbaden: VS Verlag für Sozialwissenschaften, 69-85. 
Weiner, Annette (1978). The Reproductive Model in Trobriand Society. The Australian Journal of Anthropology, 11(3), 175-186.

Welsch, Wolfgang (2010). Was ist eigentlich Transkulturalität? In: Darowska, Lucyna, Thomas Lüttenberg und Claudia Machold (Hg.). Hochschule als transkultureller Raum? Kultur, Bildung und Differenz in der Universität. Bielefeld: Transcript, 39-66.

Zehden, Maya (2017). Simantov - Jüdisches Liebesglück via Skype. Jüdische Rundschau, Juli 2017, 36. 